\title{
Aperture Field Estimation in Waveguide for Non-Sinusoidal Periodic Excitation
}

\author{
Sushrut Das, Kalidas Marandi \\ Department of Electronics Engineering, Indian School of Mines, Dhanbad, India. \\ Email: sushrut_das@yahoo.com \\ Received January $3^{\text {rd }}, 2012$; revised February $1^{\text {st }}, 2012$; accepted February $9^{\text {th }}, 2012$
}

\begin{abstract}
In this paper an attempt has been made to find the aperture field distribution in a rectangular waveguide for non-sinusoidal, periodic excitations using Multiple Cavity Modeling Technique. The excitation functions, considered, are square, trapezoidal and clipped sine wave in nature. In the present analysis these time domain excitation functions have been represented in terms of a truncated Fourier series consisting of the fundamental frequency and its higher harmonics. Within the waveguide the fundamental frequency will give rise to a dominant mode excitation whereas the higher order modes will excite dominant and higher order modes. If the higher harmonics are assumed suppressed then the waveguide is subjected only to a dominant mode excitation. Results for dominant mode reflection coefficient (magnitude), VSWR and complex transmission coefficient have been computed and compared with theoretical data. The excellent agreement between them validates the analysis.
\end{abstract}

Keywords: Waveguides; Aperture Distribution; Non-Sinusoidal Periodic Excitation; Moment Method

\section{Introduction}

Waveguide and waveguide based components are used since World War II and they are still continued to be in use. These waveguide based elements have got wide applications in ground based, air borne and ship borne radars as well as in onboard satellite application in various frequency bands ranging from $1 \mathrm{GHz}$ to $1000 \mathrm{GHz}$. The main reason for this is their high power handling capability, ability to sustain high environmental variations, ruggedness and the possibility of achieving high accuracy in fabrication. Modern radar system employs a number of waveguide components and circuit elements. Some of them are the irises, septum and windows, filters, waveguide Tee-junctions, waveguide power dividers, ortho-modal couplers and multiplexers etc. In addition to these waveguide components apertures cut in a ground plane and slots cut along the broad wall or narrow wall are extensively used in array antennas due to their large power handling capability, generation of ultra low side lobes and excellent polarization characteristics. Raw waveguides are also used for transmitting signals from one node to another in a high power microwave networks.

During post World War II, Lewin [1] and Harrington [2] gave an extensive material on the waveguides. Till then huge works have been carried out on different waveguide and waveguide based circuits on frequency domain using variational technique, Method of Moment
(MoM), Finite Difference Time Domain (FDTD) method etc. The total amount of work on these is so huge that even a brief literature survey of it will be a report of its own. However, comparatively, very few amounts of works are available on transient analysis and non-sinusoidal periodic excitations of waveguide.

In 1954 Karbowiak [3] presented the basic theory of transient propagation in waveguides. The theory was subsequently applied to the propagation of a unit step modulated carrier and propagation of pulses. Later, in 1957, Elliot [4] studied the pulse waveform degradation due to dispersion in waveguide. He derived an expression in terms of error function and Fresnel's integrals for the exit wave shape as a function of guide length, dispersion and width of the input rectangular pulse. In 1963, Saxton and Schmitt [5] published another paper on transients in large waveguide. An analytical study of the influence of moving media on the propagation of transient electromagnetic modal waves in dispersive waveguide was reported by Berger and Griemsmann [6]. The response to impulsive excitation was determined in exact closed form and was used to demonstrate the pulse distortion. In 1970 Schulsz-Dubois [7] presented a paper on Somemrfeld pre and postcursors in the context of waveguide transients. Ito [8] presented his work on dispersion of very short pulses in waveguide. Tretyakov [9], in 1994, published an evolutionary equation for the theory of wave-guides. The theory opened up new possibilities for investigating 
transient fields directly without obligatory applications of Laplace's or Fourier's transforms. In the same year an exact closed form expression for transient fields in homogeneously filled waveguides was presented by Dvorak [10]. The closed form solution, involving Bessel's function of first kind was derived from the impulse response of the waveguide. However exact, closed form solution for complex pulses was not presented. In the paper it was also demonstrated that the incomplete Lipschitz-Hankel integrals can be used to represent transient pulses in homogeneously filled waveguides. Next year, P. Srenus and B. York [11] studied the transient propagation in rectangular waveguide using elementary dispersion theory. A time domain theory of waveguide was also presented by Geyi [12].

However, most of these analyses assume a pulse or impulse excitation. No attention was paid on the analysis of waveguide circuits under non-sinusoidal, periodic excitations like square wave, trapezoidal wave, clipped sine wave, triangular wave, saw tooth wave etc. Out of these the first three have huge technical significance in high power applications like radar. This is because in Continuous Wave Doppler radar if the gain and/or the input time harmonic signal of the amplifier is/are very high then the output signal may be clipped off resulting in a clipped sinusoidal wave. Depending on the clipping level it may be approximated as a trapezoidal or even a square wave.

In this paper, a methodology for the analysis of a waveguide under non-sinusoidal periodic excitation has been carried out and the aperture field distributions in the waveguide have been plotted for different excitation.

\section{Theory}

The proposed waveguide structure has been analyzed using Multiple Cavity Modeling Technique (MCMT) [13-15]. The cavity modeling of the structure with details of different regions and fictitious magnetic currents (equivalent source to electric field) at the apertures are shown in Figure 1. The structure has three regions, namely, two waveguide regions \& one cavity regions and two interfacing apertures between them. The electric field

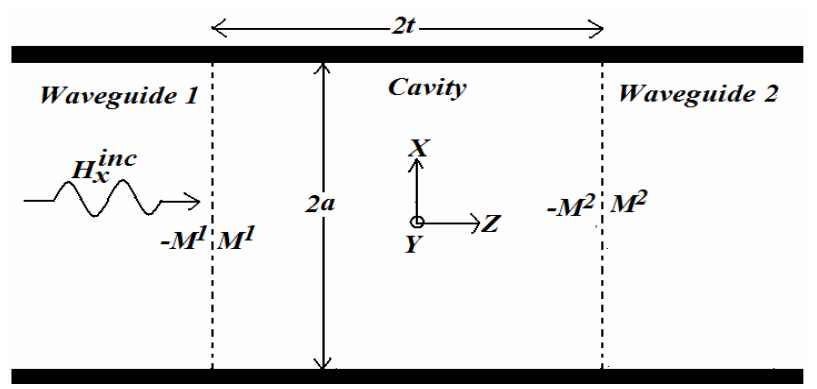

Figure 1. Details of regions and fictitious magnetic currents at the apertures. distributed on apertures (and hence the fictitious magnetic currents) are unknown and to be determined. The $\xi$ component of electric field at the $i^{\text {th }}$ aperture can be approximated in terms of unknown complex basis coefficients $E_{p q}^{i, \xi}$ and known two dimensional piecewise traingular basis function $(T(\xi))$ as [13-15]

$$
E_{\xi}^{i}=\sum_{p=1}^{M} \sum_{q=1}^{M} E_{p q}^{i, \xi} T_{p}(x) T_{q}(y) \quad \text { where } \xi=x / y
$$

and

$$
T_{v}(\xi)=\left(\begin{array}{ll}
\frac{\xi-\xi_{v-1}}{\xi_{v}-\xi_{v-1}} & \text { for } \xi_{v-1}<\xi<\xi_{v} \\
\frac{\xi_{v+1}-\xi}{\xi_{v+1}-\xi_{v}} & \text { for } \xi<\xi<\xi_{v+1}
\end{array}\right)
$$

The value of " $M$ ' in Equation (1) depends on the convergence and desired accuracy of the result. For the present analysis we have taken $M=25$. Higher the value of " $M$ ' better is the accuracy and larger is the simulation time.

Since the basis function is known we need to find the value of the basis coefficients in Equation (1) to find the aperture electric field distribution/fictitious magnetic current distribution existing at the aperture. The fictitious magnetic current $M_{\xi}^{i}$ is basically the equivalent source of the aperture electric field and is related with it by the equivalent principle.

The boundary conditions (continuity of tangential component of magnetic field across the dielectric-dielectric boundary) at the apertures can be obtained using superposition principle and can be given by

$$
\begin{aligned}
& H_{x}^{w v g 1}\left(M_{x}^{1}\right)+H_{x}^{c a v}\left(M_{x}^{1}\right)+H_{x}^{w v g 1}\left(M_{y}^{1}\right) \\
& +H_{x}^{c a v}\left(M_{y}^{1}\right)-H_{x}^{c a v}\left(M_{x}^{2}\right)-H_{x}^{c a v}\left(M_{y}^{2}\right)=2 H_{x}^{i n c} \\
& H_{y}^{w v g 1}\left(M_{x}^{1}\right)+H_{y}^{c a v}\left(M_{x}^{1}\right)+H_{y}^{w v g 1}\left(M_{y}^{1}\right) \\
& +H_{y}^{c a v}\left(M_{y}^{1}\right)-H_{y}^{c a v}\left(M_{x}^{2}\right)-H_{y}^{c a v}\left(M_{y}^{2}\right)=0 \\
& -H_{x}^{c a v}\left(M_{x}^{1}\right)-H_{x}^{c a v}\left(M_{y}^{1}\right)+H_{x}^{c a v}\left(M_{x}^{2}\right) \\
& +H_{x}^{w v g 2}\left(M_{x}^{2}\right)+H_{x}^{c a v}\left(M_{y}^{2}\right)+H_{x}^{w v g}\left(M_{y}^{2}\right)=0 \\
& -H_{y}^{c a v}\left(M_{x}^{1}\right)-H_{y}^{c a v}\left(M_{y}^{1}\right)+H_{y}^{c a v}\left(M_{x}^{2}\right) \\
& +H_{y}^{w v g 2}\left(M_{x}^{2}\right)+H_{y}^{c a v}\left(M_{y}^{2}\right)+H_{y}^{w v g 2}\left(M_{y}^{2}\right)=0
\end{aligned}
$$

The magnetic field scattered inside the cavity region due to the fictitious magnetic currents at the apertures can be determined by using cavity Green's function of the electric vector potential. The cavity Green's function has been derived by solving the Helmholtz equation for the electric vector potential for unit magnetic current source [13]. The scattered magnetic fields in the waveguide region due to the presence of the fictitious transverse 
magnetic current densities are solved by rigorous mode matching technique $[2,13]$. The incident magnetic fields are assumed to be X-directed and can be obtained by solving wave equations [16].

The tangential component of magnetic fields in the cavity region due to magnetic current in aperture " $i$ " can be expressed as

$$
\begin{aligned}
& H_{x}^{c a v}\left(M_{x}^{i}\right) \\
& =\frac{j \omega \varepsilon}{k^{2}} \sum_{p}^{M} \sum_{q}^{M} E_{p q}^{i, y} \sum_{m=0}^{\infty} \sum_{n=1}^{\infty} \frac{\varepsilon_{m} \varepsilon_{n}}{4 a b}\left[k^{2}-\left(\frac{n \pi}{2 a}\right)\right] \\
& \times \sin \left[\frac{m \pi}{2 a}(x+a)\right] \cos \left[\frac{n \pi}{2 b}(y+b)\right] \frac{\operatorname{TRIAYX}(p) \operatorname{TRIAYY}(q)}{\Gamma_{m n} \sin \left(2 \Gamma_{m n} d\right)} \\
& \times\left(\begin{array}{l}
\cos \left[\Gamma_{m n}(z-d)\right] \cos \left[\Gamma_{m n}\left(z^{\prime}+d\right)\right] \text { for } z>z^{\prime} \\
\cos \left[\Gamma_{m n}\left(z^{\prime}-d\right)\right] \cos \left[\Gamma_{m n}(z+d)\right] \text { for } z<z^{\prime}
\end{array}\right)
\end{aligned}
$$$$
H_{x}^{c a v}\left(M_{y}^{i}\right)
$$$$
=-\frac{j \omega \varepsilon}{k^{2}} \sum_{p}^{M} \sum_{q}^{M} E_{p q}^{i, x} \sum_{m=0}^{\infty} \sum_{n=1}^{\infty} \frac{\varepsilon_{m} \varepsilon_{n}}{4 a b}\left(\frac{m \pi}{2 a}\right)\left(\frac{n \pi}{2 b}\right)
$$$$
\times \sin \left[\frac{m \pi}{2 a}(x+a)\right] \cos \left[\frac{n \pi}{2 b}(y+b)\right] \frac{\operatorname{TRIAYX}(p) \operatorname{TRIAYY}(q)}{\Gamma_{m n} \sin \left(2 \Gamma_{m n} d\right)}
$$$$
\times\left(\begin{array}{ll}
\cos \left[\Gamma_{m n}(z-d)\right] \cos \left[\Gamma_{m n}\left(z^{\prime}+d\right)\right] & \text { for } z>z^{\prime} \\
\cos \left[\Gamma_{m n}\left(z^{\prime}-d\right)\right] \cos \left[\Gamma_{m n}(z+d)\right] & \text { for } z<z^{\prime}
\end{array}\right)
$$$$
H_{y}^{c a v}\left(M_{x}^{i}\right)
$$$$
=-\frac{j \omega \varepsilon}{k^{2}} \sum_{p}^{M} \sum_{q}^{M} E_{p q}^{i, y} \sum_{m=1}^{\infty} \sum_{n=0}^{\infty} \frac{\varepsilon_{m} \varepsilon_{n}}{4 a b}\left(\frac{m \pi}{2 a}\right)\left(\frac{n \pi}{2 b}\right)
$$$$
\times \cos \left[\frac{m \pi}{2 a}(x+a)\right] \sin \left[\frac{n \pi}{2 b}(y+b)\right] \frac{\operatorname{TRIAXX}(p) \operatorname{TRIAXY}(q)}{\Gamma_{m n} \sin \left(2 \Gamma_{m n} d\right)}
$$$$
\times\left(\begin{array}{ll}
\cos \left[\Gamma_{m n}(z-d)\right] \cos \left[\Gamma_{m n}\left(z^{\prime}+d\right)\right] & \text { for } z>z^{\prime} \\
\cos \left[\Gamma_{m n}\left(z^{\prime}-d\right)\right] \cos \left[\Gamma_{m n}(z+d)\right] & \text { for } z<z^{\prime}
\end{array}\right)
$$

$$
H_{y}^{c a v}\left(M_{y}^{i}\right)
$$$$
=\frac{j \omega \varepsilon}{k^{2}} \sum_{p}^{M} \sum_{q}^{M} E_{p q}^{i, x} \sum_{m=0}^{\infty} \sum_{n=1}^{\infty} \frac{\varepsilon_{m} \varepsilon_{n}}{4 a b}\left[k^{2}-\left(\frac{n \pi}{2 b}\right)\right]
$$$$
\times \cos \left[\frac{m \pi}{2 a}(x+a)\right] \sin \left[\frac{n \pi}{2 b}(y+b)\right] \frac{\operatorname{TRIAYX}(p) \operatorname{TRIAYY}(q)}{\Gamma_{m n} \sin \left(2 \Gamma_{m n} d\right)}
$$$$
\times\left(\begin{array}{ll}
\cos \left[\Gamma_{m n}(z-d)\right] \cos \left[\Gamma_{m n}\left(z^{\prime}+d\right)\right] & \text { for } z>z^{\prime} \\
\cos \left[\Gamma_{m n}\left(z^{\prime}-d\right)\right] \cos \left[\Gamma_{m n}(z+d)\right] & \text { for } z<z^{\prime}
\end{array}\right)
$$

where

$$
\begin{array}{r}
\operatorname{TRI} \xi \xi(v)=\int_{\xi_{v-1}}^{\xi_{v+1}} \sin \left[\frac{m \pi}{2 l}(\xi+l)\right] T(\xi) d \xi \\
\operatorname{TRI\zeta \xi (v)}=\int_{\xi_{v-1}}^{\xi_{v+1}} \cos \left[\frac{m \pi}{2 l}(\xi+l)\right] T(\xi) d \xi
\end{array}
$$

where $v=1,2 \cdots N ; \xi=X, \zeta=Y / \xi=Y, \zeta=X$ and $1=\mathrm{a} / \mathrm{b}$.

In Equations (7)-(10), $2 a$ is the guide width, $2 b$ is the guide height, $\varepsilon_{m}$ and $\varepsilon_{n}$ are the Neumann's function, $2 d$ is the cavity thickness/length of the waveguide, $\Gamma_{m n}$ is the propagation constant for the $(m, n)$ mode. The rest have their usual meanings.

The tangential component of magnetic fields in the waveguide region due to magnetic current in aperture " $i$ " can be expressed as

$$
\begin{aligned}
& H_{x}^{w v g}\left(M_{x}^{i}\right)=-\sum_{p}^{M} \sum_{q}^{M} E_{p q}^{i, y} \\
& \times \sum_{m=1}^{\infty} \sum_{n=0}^{\infty}\left[Y_{m n}^{e}\left\{C_{m n}^{e}\left(\frac{m \pi}{2 a}\right)\right\}^{2}+Y_{m n}^{m}\left\{C_{m n}^{m}\left(\frac{n \pi}{2 b}\right)^{2}\right\}\right] \\
& \times \sin \left[\frac{m \pi}{2 a}(x+a)\right] \cos \left[\frac{n \pi}{2 b}(y+b)\right] \operatorname{TRIAXX}(p) \operatorname{TRIAXY}(q)
\end{aligned}
$$

$$
H_{x}^{w v g}\left(M_{y}^{i}\right)=\sum_{p}^{M} \sum_{q}^{M} E_{p q}^{i, x}
$$

$$
\begin{aligned}
& \times \sum_{m=1}^{\infty} \sum_{n=1}^{\infty}\left[Y_{m n}^{e}\left(C_{m n}^{e}\right)^{2}-Y_{m n}^{m}\left(C_{m n}^{m}\right)^{2}\right]\left(\frac{m \pi}{2 a}\right)\left(\frac{n \pi}{2 b}\right) \\
& \times \sin \left[\frac{m \pi}{2 a}(x+a)\right] \cos \left[\frac{n \pi}{2 b}(y+b)\right] \operatorname{TRIAYX}(p) \operatorname{TRIAYY}(q)
\end{aligned}
$$

$$
H_{y}^{w v g}\left(M_{x}^{i}\right)=-\sum_{p}^{M} \sum_{q}^{M} E_{p q}^{i, y}
$$

$$
\times \sum_{m=1}^{\infty} \sum_{n=1}^{\infty}\left[Y_{m n}^{e}\left(C_{m n}^{e}\right)^{2}-Y_{m n}^{m}\left(C_{m n}^{m}\right)^{2}\right]\left(\frac{m \pi}{2 a}\right)\left(\frac{n \pi}{2 b}\right)
$$$$
\times \cos \left[\frac{m \pi}{2 a}(x+a)\right] \sin \left[\frac{n \pi}{2 b}(y+b)\right] \operatorname{TRIAXX}(p) \operatorname{TRIAXY}(q)
$$

$$
H_{y}^{w v g}\left(M_{y}^{i}\right)=\sum_{p}^{M} \sum_{q}^{M} E_{p q}^{i, x}
$$$$
\times \sum_{m=0}^{\infty} \sum_{n=1}^{\infty}\left[Y_{m n}^{e}\left\{C_{m n}^{e}\left(\frac{n \pi}{2 b}\right)\right\}^{2}+Y_{m n}^{m}\left\{C_{m n}^{m}\left(\frac{m \pi}{2 a}\right)^{2}\right\}\right]
$$$$
\times \cos \left[\frac{m \pi}{2 a}(x+a)\right] \sin \left[\frac{n \pi}{2 b}(y+b)\right] \operatorname{TRIAYX}(p) \operatorname{TRIAYY}(q)
$$

where 


$$
\begin{gathered}
C_{m n}^{e}=\frac{1}{\pi} \sqrt{a b \varepsilon_{m} \varepsilon_{n} /\left\{(m b)^{2}+(n a)^{2}\right\}} \\
C_{m n}^{m}=\frac{2}{\pi} \sqrt{a b /\left\{(m b)^{2}+(n a)^{2}\right\}}
\end{gathered}
$$

The incident fields will be composed of a number TE and TM modes that are generated at the feed point discontinuity and are supported by the waveguide and can be written as

$$
\begin{aligned}
H_{x, T E}^{i n c} & =H_{0} \sum_{m} \sum_{n} \frac{\Gamma_{m n}}{h^{2}}\left(\frac{m \pi}{2 a}\right) \\
& \times \sin \left[\frac{m \pi}{2 a}(x+a)\right] \cos \left[\frac{n \pi}{2 b}(y+b)\right] \\
H_{x, T M}^{i n c} & =E_{0} \sum_{m} \sum_{n} \frac{j \omega \varepsilon}{h^{2}}\left(\frac{n \pi}{2 b}\right) \\
& \times \sin \left[\frac{m \pi}{2 a}(x+a)\right] \cos \left[\frac{n \pi}{2 b}(y+b)\right]
\end{aligned}
$$

provided the incident field is assumed to be X-directed. If we further assume that the geometric distribution of the field source is such that the excitation is a linear combination of the supported modes then the amplitude $H_{0}$ and $E_{0}$ of Equations (19) and (20) can be obtained from the Fourier coefficients of the input signal. If $\tau$ be the rise and fall time and $T$ be the time period then the Fourier coefficients for square, trapezoidal and clipped sine wave respectively can be given by (for all the cases $a_{0}$ and $a_{n^{\prime}}$ is zero)

$$
\begin{gathered}
b_{n^{\prime}, \text { Square }}=4 / n^{\prime} \pi \\
b_{n^{\prime}, \text { Trapezoidal }}=\left\{4 \sin \left(n^{\prime} \omega \tau\right)\left[1-\cos \left(n^{\prime} \omega T / 2\right)\right]\right\} /\left(n^{\prime 2} \omega^{2} T \tau\right) \\
b_{n^{\prime}, \text { Clipped Sinusoidal }}=\frac{2}{T}\left\{\frac{A \tau}{2} \operatorname{sinc}\left[\omega \tau\left(n^{\prime}-1\right)\right]\right. \\
\times\left\{1+2 \cos \left[\frac{\omega T}{2}\left(n^{\prime}-1\right)\right]+\cos \left[\omega T\left(n^{\prime}-1\right)\right]\right\} \\
-\frac{A \tau}{2} \operatorname{sinc}\left[\omega \tau\left(n^{\prime}+1\right)\right]\left\{1+2 \cos \left[\frac{\omega T}{2}\left(n^{\prime}+1\right)\right]\right. \\
\left.\left.+\cos \left[\omega T\left(n^{\prime}+1\right)\right]\right\}+\frac{2}{n^{\prime} \omega} \cos \left(n^{\prime} \omega \tau\right)\left[1-\cos \left(\frac{n^{\prime} \omega T}{2}\right)\right]\right\}
\end{gathered}
$$

Solving the boundary conditions, provided in Equations (3)-(6) by Galerkin's specialization of Method of moment, where weighting function is same as expressed in equation Equations (1) and (2), the aperture electric field distribution can be obtained.

From the aperture electric field distribution, obtained using the present theory, we can easily calculate the com- plex reflection $(\Gamma)$ and transmission $(T)$ coefficients using the following equations:

$$
\begin{aligned}
\Gamma= & -1 \\
& +\left.\left.\frac{1}{2 a b} Y_{10} \sum_{p=1}^{M} \sum_{q=1}^{M} E_{p q}^{1, x} \operatorname{TRIAXX}(p)\right|_{m=1} \operatorname{TRIAXY}(q)\right|_{n=0}
\end{aligned}
$$

and

$$
T=\left.\left.\frac{1}{2 a b} Y_{10} \sum_{p=1}^{M} \sum_{q=1}^{M} E_{p q}^{2, x} \operatorname{TRIAXX}(p)\right|_{m=1} \operatorname{TRIAXY}(q)\right|_{n=0}
$$

\section{Results and Discussion}

The electric field distribution at apertures of waveguide for square, trapezoidal and clipped sine excitations have been normalized with respect to the maximum aperture field strength and are shown in Figures 2-4 respectively. The figures show that the aperture electric field distributions are not purely sinusoidal. This is due to the presence of the higher order modes in the guide. However as the excitation changes from square to clipped sine wave it become more sinusoidal. This is because of the rapid convergence of the Fourier series and hence presence of lesser number of modes.

If we set the rise/fall time of the broken sine wave equal to $T / 4$ then the excitation function changes to a sinusoid excitation. The dominant mode reflection coefficient (magnitude), VSWR and transmission coefficient for such case have been computed using Equations (24) and (25) and compared with the available theoretical data in Figure 5. The theoretical data for $\left|\mathrm{S}_{11}\right|$, VSWR and $\left|\mathrm{S}_{21}\right|$ are not shown in Figure 5 as they are well known to be constant at 0,1 and 1 respectively. Theoretical value of the phase of the transmission coefficient is equal to $4 \pi t / \lambda_{g}$ where $\lambda_{g}$ is the guided wavelength.

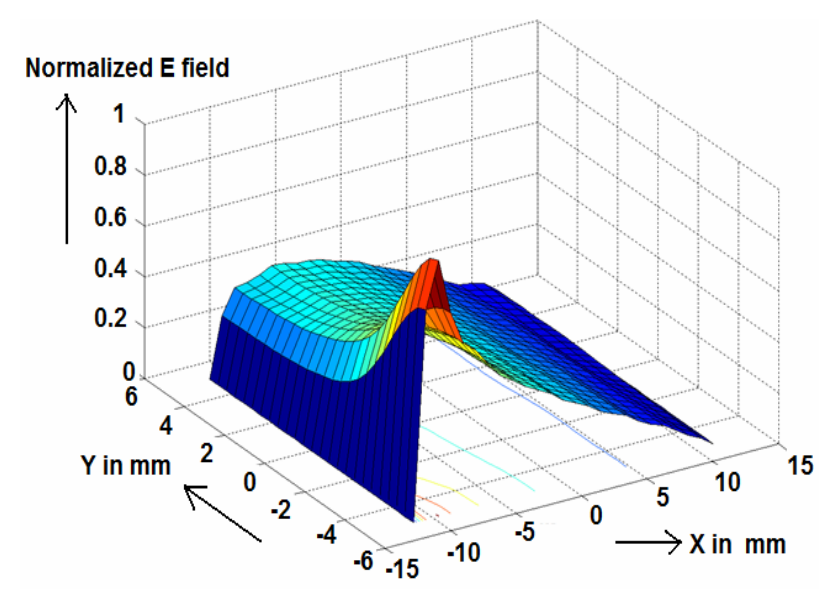

Figure 2. Normalized electric field distribution in waveguide for square wave excitation. 


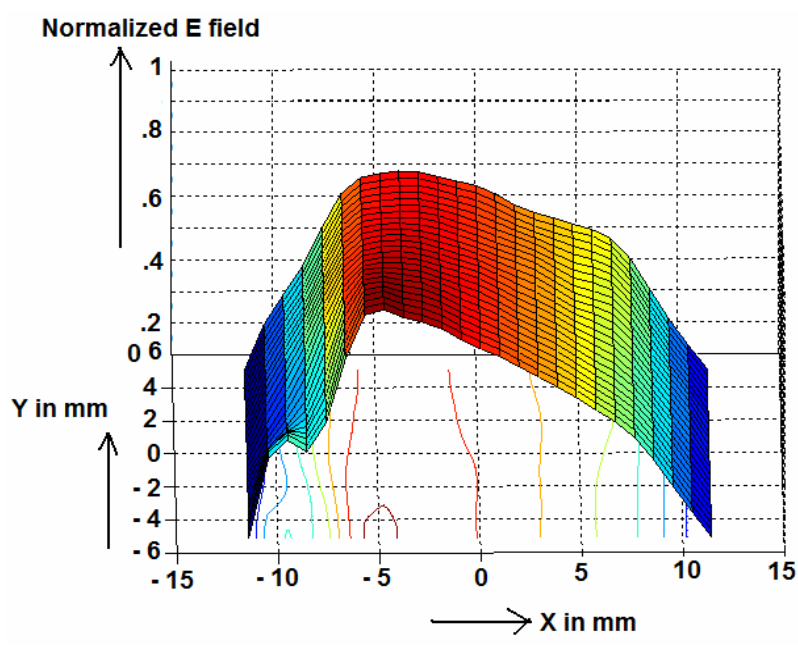

Figure 3. Normalized electric field distribution in waveguide for trapezoid wave excitation.

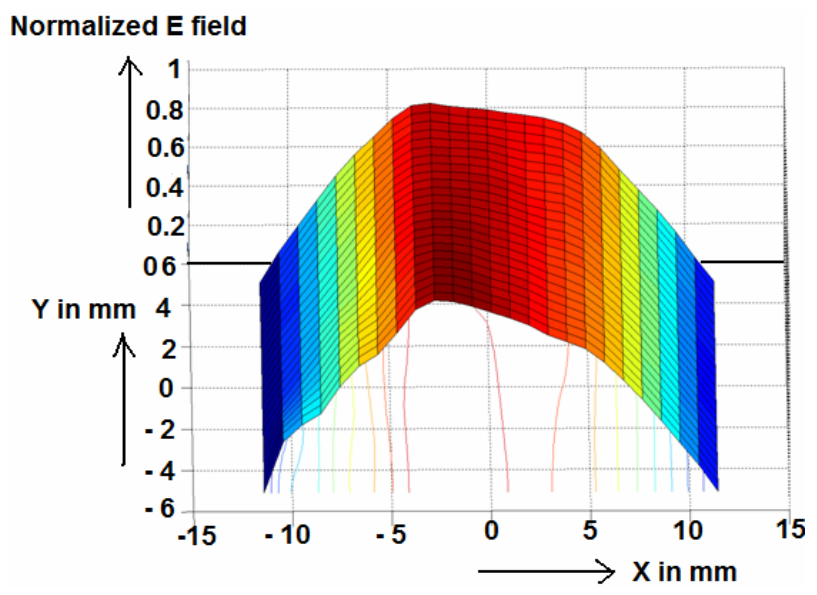

Figure 4. Normalized electric field distribution in waveguide for clipped sine wave excitation.

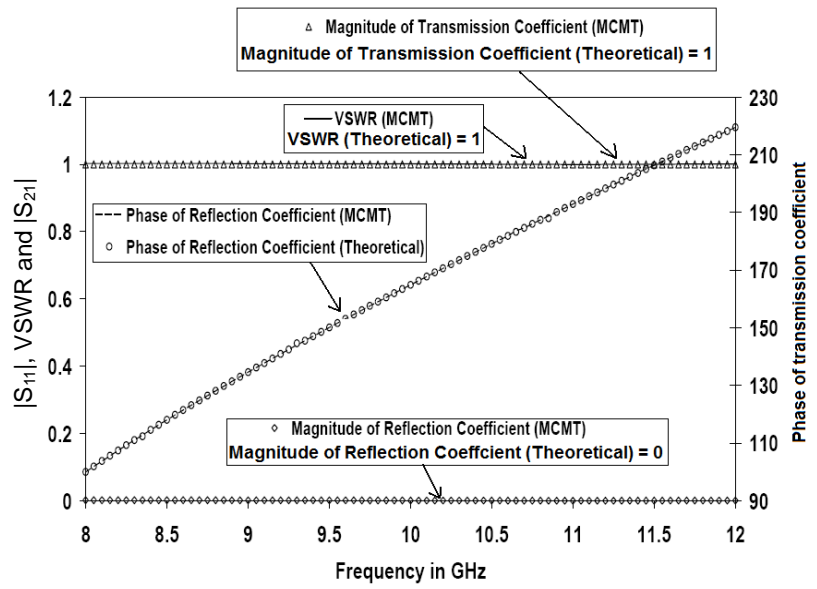

Figure 5. Plot of reflection coefficient (magnitude), VSWR and transmission coefficient. The theoretical value of the magnitude of reflection coefficient $=0$, Magnitude of transmission coefficient $=1$ and VSWR $=1$ are standard and hence not shown in the graph.

\section{Conclusions}

The paper presents a methodology to find the aperture electric field distribution in the waveguide for square, trapezoidal and clipped sine wave excitation. The methodology is general and can be extended to other waveguide circuits excited by any deterministic and periodic waveform that can be extended in Fourier series.

It should be that noted our main objective of the present work is to propose a methodology to find the aperture field distribution in a waveguide circuit for non sinusoidal periodic excitation. This is because if we can find the aperture field distribution then from it we can calculate the complete characteristic of the circuit under the given excitation.

The proposed theory can be extended for any arbitrary not periodic waveform also. However for such cases we must know the strengths of the individual mode existing within the circuit.

The proposed theory is also applicable to other rectangular waveguide based circuits under periodic/nonperiodic non-sinusoidal excitations.

\section{Acknowledgements}

The work is supported by Department of Science and Technology, Government of India and the authors wish to express their gratitude for this support.

\section{REFERENCES}

[1] L. Lewin, "Advanced Theory of Waveguide," Iliffe, 1951.

[2] R. F. Harrington, "Time-Harmonic Electromagnetic Fields," IEEE Press Classic Reissue, John Wiley \& Sons, Inc., New York, 2001.

[3] A. E. Karbowiak, "Propagation of Transients in Waveguides," Proceedings of IEE_Part C: Monographs, Vol. 104, No. 6, 1957, pp. 339-348.

[4] R. S. Elliot, "Pulse Waveform Degradation Due to Dispersion in Waveguide," IRE Transactions on Microwave Theory and Technique, Vol. 5, No. 4, 1957, pp. 254-257. doi:10.1109/TMTT.1957.1125161

[5] W. A. Saxton and H. J. Schmitt, "Transients in Large Waveguide," Proceedings of the IEEE, Vol. 51, No. 2, 1963, pp. 405-406. doi:10.1109/PROC.1963.1829

[6] H. Berger and J. W. E. Griemsmann, "Transient Electromagnetic Guided Wave Propagation in Moving Media," IEEE Transactions on Microwave Theory and Techniques, Vol. 16, No. 10, 1968, pp. 842-849. doi:10.1109/TMTT.1968.1126801

[7] E. O. Schulz-Dubois, "Sommerfeld Pre- and Postcursors in the Context of Waveguide Transients," IEEE Transactions on Microwave Theory and Techniques, Vol. 18, No. 8, 1970, pp. 455-460. doi:10.1109/TMTT.1970.1127268

[8] M. Ito, "Dispersion of Very Short Microwave Pulses in Waveguide," IEEE Transactions on Microwave Theory 
and Techniques, Vol. 13, No. 3, 1965, pp. 357-364. doi:10.1109/TMTT.1965.1126000

[9] O. A. Tretyakov, "Evolutionary Equations for the Theory of Waveguides," IEEE Antennas and propagation Society International Symposium, Vol. 3, 1994, pp. 1973-1976.

[10] S. L. Dvorak, "Exact Closed-Form Expression for Transient Fields in Homogeneously Filled Waveguides," IEEE Transactions on Microwave Theory and Techniques, Vol. 42, No. 11, 1994, pp. 2164-2170. doi:10.1109/22.330133

[11] P. Stenius and B. York, "On the Propagation of Transients in Waveguides," IEEE Antennas and Propagation Magazine, Vol. 37, No. 2, 1995, pp. 39-44. doi:10.1109/74.382337

[12] G. Y. Wen, "A Time Domain Theory of Waveguide," Progress in Electromagnetic Research, PIER 59, 2006, pp. 267-297. doi:10.2528/PIER05102102

[13] A. Vengadarajan, "Multiple Cavity Modeling Technique for Solving Aperture Coupled Waveguide Junctions," Ph.D. Dissertation, Indian Institute of Technology, Kharagpur, 1999.

[14] S. Das, "Analysis of Rectangular Waveguide Based Passive Devices and Antennas Using Multiple Cavity Modeling Technique," Ph.D. Dissertation, Indian Institute of Technology, Kharagpur, 2007.

[15] S. Das, "A Novel Modeling Technique to Solve a Class of Rectangular Waveguide Based Circuits and Radiators," Progress in Electromagnetic Research, PIER 61, 2006, pp. 231-252. doi:10.2528/PIER06010302

[16] D. M. Pozar, "Microwave Engineering," 2nd Edition, John Wiley \& Sons (Asia) Pte. Ltd., Singapore, 2004. 